\title{
Laser generation without inversion on the fine levels of the helium atom
}

\author{
A. G. Petrashen, N. V. Sytenko \\ ITMO University, 197101, Saint Petersburg, Russia \\ apetrashen@mail.ru
}

PACS 32.80.Qk

DOI 10.17586/2220-8054-2016-7-2-384-393

By using the example of transfer between fine structure levels in the helium atom, the possibility of laser generation without inversion has been studied.

Keywords: coherent exitation, dehsity matrix evolution, dark states.

Received: 20 November 2015

\section{Introduction}

The possibility of lasing without inversion (LWI) was first postulated by Kocharovskaya and Harris [1,2]. In the latter of these works, the assumption of LWI viability had been based on a comparison of the absorption and transmission coefficients of a three-level system, which is excited according to $V$-scheme, whereas in previous works, it was shown that, for certain parameter values for a system which is excited according to $\Lambda$-scheme, the laser generation without inversion is possible. Furthermore, in our opinion, the work of Imamoglu and Harris [3], must be highlighted. In this work, issued shortly after those two mentioned above, the opinion was first expressed that LWI could be produced using the absorption that disappeared within one of the $\Lambda$-scheme arms, whereas within another arm, excitation is performed by means of a strong EM-field (phenomenon of electromagnetic induced transparency (EIT)) [4]. In the subsequent works, the possibility for LWI, in principle, has been widely discussed (see, e.g. [4-9]).

However, Zibrov et al. [11] were the first to experimentally observe the LWI phenomenon, where a set of four levels for the hyperfine atomic structure of ${ }^{87} \mathrm{Rb}$ was considered: two hyperfine levels for the ground ${ }^{2} \mathrm{~S}_{1 / 2}$ state, as well as two analogous levels for the first excited ${ }^{2} \mathrm{P}_{1 / 2}$ state. The strong-coupled and weak probe fields were bound according to the $V$-scheme one superfine level of the ground state with two superfine levels of the first exited state. In the article, the frequency range at which the absorption coefficient becomes negative, i.e. lasing generation occurs, was determined both theoretically (by means of numerical solution of the Liouville equation for the density matrix) and experimentally.

Work [12] is similar to one just mentioned, with the difference being that LWI was experimentally implemented using sodium atoms in the framework of the $\Lambda$-scheme, formed by two hyperfine levels of the ground state ${ }^{2} \mathrm{~S}_{1 / 2}$ and level $F=1$ of the first excited state ${ }^{2} \mathrm{P}_{1 / 2}$ of this atom. In this work, the strong coupling field acts between the $F=2$ levels of the ${ }^{2} \mathrm{~S}_{1 / 2}$ state and the $F=1$ level of the ${ }^{2} \mathrm{P}_{1 / 2}$ state. This field, in combination with very weak probe field, connecting levels of the hyperfine structure with $F=1$ of the ${ }^{2} \mathrm{~S}_{1 / 2}$ and ${ }^{2} \mathrm{P}_{1 / 2}$ states leads to the appearance of EIT resonance. Additionally, it was experimentally shown that at a frequency close to where EIT occurs, amplification of the probe signal is observed. Subsequently, the system has been positioned inside an annular resonator, and laser oscillation was observed within the system, being provided by vacuum fluctuations. 
In this work, the possibility of laser generation without inversion (LWI) within a multilevel system that is positioned inside a high- $Q$ annular resonator will be considered. The subject being dealt with here is closely allied with work [13], where are a non-inversion superreliance impulse was observed for fine levels of the helium atom.

\section{The statement of the problem}

In this work, radiation emitted from $3^{3} \mathrm{~S}_{1}$ state (level "b") of isotope ${ }^{4} \mathrm{He}$, whose coherent excitation is performed according to the $\Lambda$-scheme from levels $2^{3} \mathrm{P}_{2}$ and $2^{3} \mathrm{P}_{1}$ (hereafter referred to as, levels "a" and "c") of this atom (see Fig. 1), will be considered. It should be noted that the first of those two lower states could be referred to as metastable ones [16], and hereupon a considerable population could be created at this level, for example, by means of an impact from the ground state. In the framework of such an excitation, the fine bond is broken, and the efficiency of population induction for the $2^{3} \mathrm{P}$ state could be described as follows:

$$
\sigma_{j, m, j^{\prime}, m^{\prime}}=\frac{1}{2 j+1} A^{(0)}\left(j, j^{\prime}, s\right)+\alpha \sum_{q}(-1)^{j^{\prime}+m^{\prime}}\left[\begin{array}{ccc}
j & j^{\prime} & 2 \\
m & -m^{\prime} & q
\end{array}\right] D_{0, q}^{(2)}(\theta) A^{(2)}\left(j, j^{\prime}, l, s\right),
$$

where $j$ and $s$ depict total electron and spin moments, parameter $\alpha$ characterizes an anisotropy of impact excitation. As an example, this value may be set as a ratio of induced orbital alignment to a population [14]: $\alpha=T_{0}^{2} / T_{0}^{0}$, and, finally, $D_{m, m^{\prime}}^{(2)}(\theta)$ - elements of three-dimensional rotational matrix [15]. The quantities $A^{(k)}$ in this expression depend only on the kinematics of momentum vectorial addition:

$$
A^{(k)}\left(j, j^{\prime}, l, s\right)=(-1)^{j^{\prime}+s+k+l} \sqrt{(2 j+1)\left(2 j^{\prime}+1\right)}\left\{\begin{array}{lll}
l & l & k \\
j & j^{\prime} & s
\end{array}\right\} .
$$

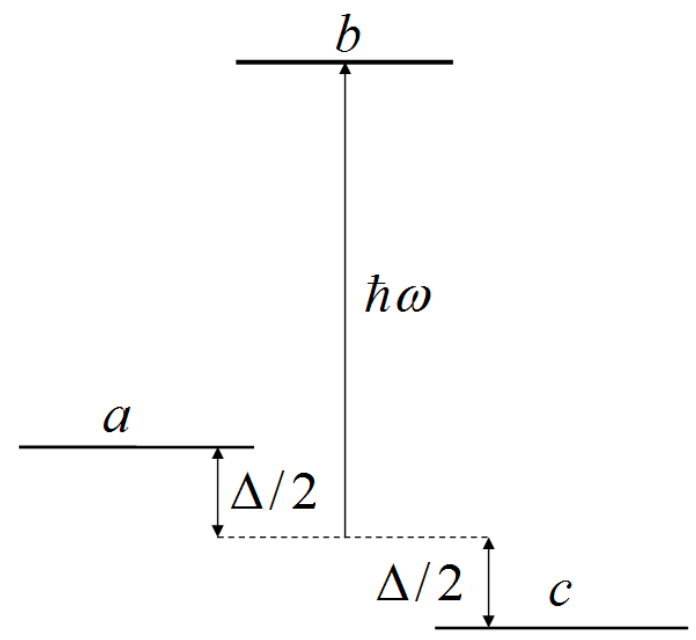

FIG. 1. The excitation scheme form:

In this work, we will assume, that the Hamilton operator of the atomic system takes the

$$
\hat{H}=\hat{H}_{0}+\hat{V},
$$

where $H_{0}$ is the Hamiltonian operator of a free helium atom, while operator $\hat{V}$ describes the excitation process of level $j=1$ (hereafter, of the level "b") of $3{ }^{3} \mathrm{~S}_{1}$ state of the aforementioned atom. In regard to the excitation process, we will assume that in both arms, $a b$ and $c b$, excitation is initiated by means of light polarized linearly along the $O Z$-axis laboratory frame of reference 
(this axis coincides with the direction of propagation for the exiting radiation), at frequencies $\omega-\delta_{a} / \hbar$ and $\omega+\delta_{c} / \hbar$, respectively. The wave functions of all states have been constructed according to general addition rules for angular momenta, whereas energy parameters of those functions have been chosen according to the quantum defect method:

$$
\begin{aligned}
\left(W_{a b}\right)_{M M_{1}} & =\sum_{m, q}\left[\begin{array}{ccc}
1 & 1 & 1 \\
m & M_{1} & M
\end{array}\right]\left[\begin{array}{ccc}
0 & 1 & 1 \\
0 & q & m
\end{array}\right]\left(e_{a b}\right)_{q} \cos \left(\omega+\delta_{a} / \hbar\right), \\
\left(W_{c b}\right)_{M M_{1}} & =\sum_{m, q}\left[\begin{array}{ccc}
1 & 1 & 2 \\
m & M_{1} & M
\end{array}\right]\left[\begin{array}{ccc}
0 & 1 & 1 \\
0 & q & m
\end{array}\right]\left(e_{c b}\right)_{q} \cos \left(\omega+\delta_{c} / \hbar\right),
\end{aligned}
$$

where $e_{q}$ is the path of the light polarization in some arm of the $\Lambda$-scheme.

Exciting electric field $\vec{E}(t)$ causes medium polarization that is characterized by the polarization vector $\vec{P}$, whose quantity is proportional to the density of the atoms $n_{0}$ and mean value of the dipole moment operator $\hat{d}$, calculated with the density correlation matrix:

$$
\vec{P}=-n_{0} \Im\left(\left[\operatorname{Sp}\left(\rho_{a b} \vec{d}_{a b}\right)\right]+\left[\operatorname{Sp}\left(\rho_{c b} \vec{d}_{c b}\right)\right]\right) \text {. }
$$

From the last expression, one can see that the medium polarization, as a result of excitation, is defined by the density correlation matrix, and, therefore, depends on its variation. In turn, the right side of Liouville equation that describes the density matrix evolution, depends on Rabi frequency, which is proportional to the electric field value. Therefore, mutual variation of the density matrix and of the induced electric field could be described by the following system of self-consistent equations:

$$
\begin{aligned}
\frac{d}{d t} \widetilde{\rho}_{a a}(t) & =\Gamma \widetilde{\rho}_{b b}(t)-\frac{i}{\hbar}\left(V_{a b}(t) \widetilde{\rho}_{b a}(t)-\widetilde{\rho}_{a b}(t) V_{b a}(t)\right), \\
\frac{d}{d t} \widetilde{\rho}_{b b}(t) & =-\Gamma \widetilde{\rho}_{b b}(t)-\frac{i}{\hbar}\left(V_{b a}(t) \widetilde{\rho}_{a b}(t)-\widetilde{\rho}_{b a}(t) V_{a b}(t)\right)-\frac{i}{\hbar}\left(V_{b c}(t) \widetilde{\rho}_{c b}(t)-\widetilde{\rho}_{b c}(t) V_{c b}(t)\right), \\
\frac{d}{d t} \widetilde{\rho}_{c c}(t) & =\Gamma \widetilde{\rho}_{b b}(t)-\frac{i}{\hbar}\left(V_{c b}(t) \widetilde{\rho}_{b c}(t)-\widetilde{\rho}_{c b}(t) V_{b c}(t)\right), \\
\frac{d}{d t} \widetilde{\rho}_{a b}(t) & =-\frac{\Gamma}{2} \widetilde{\rho}_{a b}(t)-\frac{i}{\hbar}\left(E_{a}-E_{b}\right) \widetilde{\rho}_{a b}(t)-\frac{i}{\hbar}\left(V_{a b}(t) \widetilde{\rho}_{b b}(t)-\widetilde{\rho}_{a a}(t) V_{a b}(t)\right), \\
\frac{d}{d t} \widetilde{\rho}_{c b}(t) & =-\frac{\Gamma}{2} \widetilde{\rho}_{c b}(t)-\frac{i}{\hbar}\left(E_{c}-E_{b}\right) \widetilde{\rho}_{c b}(t)-\frac{i}{\hbar}\left(V_{c b}(t) \widetilde{\rho}_{b b}(t)-\widetilde{\rho}_{c c}(t) V_{c b}(t)\right), \\
\widetilde{\rho}_{b a}(t) & =\widetilde{\rho}_{a b}^{\dagger}(t) ; \quad \widetilde{\rho}_{b c}(t)=\widetilde{\rho}_{c b}^{\dagger}(t), \\
\nabla^{2} \vec{E} & -\frac{1}{c^{2}} \frac{\partial^{2}}{\partial t^{2}} \vec{E}=\frac{4 \pi}{c^{2}} \frac{\partial^{2}}{\partial t^{2}} \vec{P}
\end{aligned}
$$

where the matrix $\Gamma$ describes the relaxation process, $E_{a}$ and $E_{c}$ are the energies for the lower levels "a" and "c" respectively, $E_{b}$ is the energy of the upper $3{ }^{3} \mathrm{~S}_{1}$ state. Solution of such systems is usually attempted using the rotating wave approximation, which means, that in system (6), all terms that oscillate with the frequency $\omega$ of the exciting field should be neglected. The higher order derivatives of slowly-varying summands should also be neglected. To separate such terms in the system, it is convenient to introduce density matrices $\rho_{i, j}(t)(i, j=a, b, c)$ connected with previously used similar matrices, by means of the relations: $\rho_{i i}(t)=\widetilde{\rho}_{i i}(t)$ $(i=a, b, c), \rho_{b, i}(t)=\widetilde{\rho}_{b, i}(t) \exp \left(-i\left(E_{b}-E_{i}\right) t\right), i=a, c, \rho_{i, j}(t)=\rho_{j, i}(t)^{\dagger}$. In regard to the last equation, to separate rapidly oscillating summands, field strength and field polarization vectors should be solved in the following manner: $E=\mathcal{E}(x, t) e^{i(\omega t+k x)}+\mathcal{E}^{*}(x, t) e^{-i(\omega t+k x)}$ and 
$P=\mathcal{P}(x, t) e^{i(\omega t+k x)}+\mathcal{P}^{*}(x, t) e^{-i(\omega t+k x)}$, where $\mathcal{E}(x, t)=\Re \mathcal{E}(x, t)+i \Im \mathcal{E}(x, t)$ and $\mathcal{P}(x, t)=$ $\Re \mathcal{P}(x, t)+i \Im \mathcal{P}(x, t)$. As a result of such substitutions, the last equation of system (6) will be reduced to a pair of first-order equations that links the real and imaginary parts of field strength and polarization vectors:

$$
\begin{aligned}
& \frac{d}{d t}(\Im \mathcal{E}(x, t))+c \frac{d}{d x}(\Im \mathcal{E}(x, t))=-2 \pi \omega \Re(\mathcal{P}(x, t)), \\
& \frac{d}{d t}(\Re \mathcal{E}(x, t))+c \frac{d}{d x}(\Re \mathcal{E}(x, t))=2 \pi \omega \Im(\mathcal{P}(x, t)) .
\end{aligned}
$$

Hereafter, we will assume the polarization field to be independent from the spatial coordinates. Next, we introduce the dimensionless Rabi frequency $\Omega_{R}(t)$, which is linked to the polarization vector by the relation $\Omega_{R}(t)=\mathcal{E}(t) \tau_{0} e a_{0} / \hbar$, where $\tau_{0}=36 \cdot 10^{-9} \mathrm{~s}$ [16] is the time of life of excited state $3^{3} \mathrm{~S}_{1}$, and introduce dimensionless time $\tau=t / \tau_{0}$, and, additionally, assuming that functions $\mathcal{P}$ and $\mathcal{E}$ vary slowly with time, and that the system is positioned inside an annular resonator, such that one of the eigenfrequencies coincides with the transition frequency between the "centers of gravity" for the upper and lower multiples, then, for system (8), one can write:

$$
\begin{aligned}
\frac{d}{d \tau} \rho_{a a}(\tau)= & \Gamma_{a} \rho_{b b}(\tau)+\frac{i \Omega_{R}}{2}\left[\rho_{a b}(\tau) W_{b a}(\tau) / z_{a}-W_{a b}(\tau) \rho_{b a}(\tau) z_{a}\right], \\
\frac{d}{d \tau} \rho_{c c}(\tau)= & \Gamma_{c} \rho_{b b}(\tau)+\frac{i \Omega_{R}}{2}\left[\rho_{c b}(\tau) W_{b c}(\tau) z_{c}-W_{c b}(\tau) \rho_{b c}(\tau) / z_{c}\right], \\
\frac{d}{d \tau} \rho_{b b}(\tau)= & -\Gamma \rho_{b b}(\tau)-\frac{i \Omega_{R}}{2}\left[W_{b a} \rho_{a b}(\tau) / z_{a}-\frac{i}{2 \hbar} \rho_{b a}(\tau) W_{a b} z_{a}\right]- \\
& \frac{i \Omega_{R}}{2}\left[W_{b c} \rho_{c b}(\tau) z_{c} c-\frac{i}{2 \hbar} \rho_{b c}(\tau) W_{c b} / z_{c}\right], \\
\frac{d}{d \tau} \rho_{a b}(\tau)= & -\frac{\Gamma}{2} \rho_{a b}(\tau)+\frac{i \Omega_{R}}{2}\left[\rho_{a a}(\tau) W_{a b} z_{a}-\frac{i}{2 \hbar} W_{a b} \rho_{b b}(\tau) z_{a}\right]+\frac{i \Omega_{R}}{2} \rho_{a c}(\tau) W_{c b} / z_{a}, \\
\frac{d}{d \tau} \rho_{c b}(\tau)= & -\frac{\Gamma}{2} \rho_{c b}(\tau)-\frac{i \Omega_{R}}{2}\left[W_{c b} \rho_{b b}(\tau) / z_{c}+\rho_{c c}(\tau) W_{c b} / z_{c}\right]+\frac{i \Omega_{R}}{2} \rho_{c a}(\tau) W_{a b} z_{a}, \\
\frac{d}{d \tau} \Omega_{R}= & -\Omega_{M}^{2} i\left(\operatorname{Sp}\left(\rho_{a b} d_{z}\right)+\operatorname{Sp}\left(\rho_{b c} d_{z}\right)\right), \\
\rho_{b a}(t)= & \rho_{a b}^{\dagger}(t) ; \quad \rho_{b c}(t)=\rho_{c b}^{\dagger}(t),
\end{aligned}
$$

where $z_{a}=\exp \left(i \tau\left(\frac{\Delta}{2 \hbar}+\delta_{a}\right)\right)$ and $z_{c}=\exp \left(i \tau\left(\frac{\Delta}{2 \hbar}-\delta_{c}\right)\right)$, and $\Omega_{M}$ is the dimensionless frequency of the field oscillation amplitude $\Omega_{M}=\sqrt{4 \pi n_{0}\left(\frac{e a_{0}}{\hbar}\right)^{2} \hbar \omega \frac{\langle r\rangle}{a_{0}}}$, which depends on $\langle r\rangle$ - dimensionless main value of operator $\hat{r}$ for the $3{ }^{3} S_{1}$ state. The substitution of values for the fundamental constants in the last relation leads to the relation $\Omega_{M}=5 \cdot 1610^{-4} \sqrt{n_{0}}$, where $n_{0}$ - density of the particulars.

\section{The approximate solution of the Liouville equation}

According to the fact, that the coupling field, which is acting in the "bc" arm of $\Lambda$-scheme, is considered strong relative to the field acting in the "ba" arm, a solution for system (9) could be found by means of perturbation theory, in the framework of initial conditions 
$\rho_{a a}(0)=\rho_{a a}^{(0)}, \rho_{c c}(0)=\rho_{c c}^{(0)}$ and $\rho_{b b}(0)=0$, where $\rho_{c c}(0)$ and $\rho_{b b}(0)$ are determined by relation (1). Large values for the coupling field lead to the segmentation of them considered systems splitting into blocks, the first of which associates the density correlation matrices $\rho_{a b}(t)$ and $\rho_{a c}(t)$ :

$$
\begin{aligned}
\frac{d}{d t} \rho_{a b}(t) & =-\frac{\gamma_{a b}}{2} \rho_{a b}(t)+\frac{i}{2} \rho_{a a}^{(0)} W_{a b} e^{i t\left(\delta_{a}+\Delta / 2 \hbar\right)}+\frac{i}{2} \rho_{a c}(t) W_{c b} e^{-i t\left(-\delta_{c}+\Delta / 2 \hbar\right)} \\
\frac{d}{d t} \rho_{a c}(t) & =-\frac{\gamma_{a c}}{2} \rho_{a c}(t)+\frac{i}{2} \rho_{a b}(t) W_{b c} e^{i t\left(-\delta_{c}+\Delta / 2 \hbar\right)}
\end{aligned}
$$

Now, it is pertinent to introduce matrix $x_{a c}(t)$ and $x_{a b}(t)$ according to expression:

$$
\rho_{a c}(t)=x_{a c}(t) e^{-\gamma_{a c} t / 2} ; \quad \rho_{a b}(t)=x_{a b}(t) e^{-\gamma_{a b} t / 2},
$$

then, according to the second equation of system (9), for matrix $x_{a c}(t)$, one can obtain that:

$$
\frac{d}{d t} x_{a c}(t) W_{c b}=\frac{1}{2} i \exp \left(\frac{i \Delta+\left(\gamma_{a c}-\gamma_{a b}-2 i \delta_{c}\right) \hbar}{2 \hbar} t\right) x_{a b}(t) W_{b c} W_{c b} .
$$

Then, having differentiated the first of equations (9) and substituting it into expression (11), the equation for the $x_{a c}(t)$ matrix may be obtained:

$$
\begin{aligned}
\frac{d^{2}}{d t^{2}} x_{a b}(t) & +\frac{(i \Delta)+\left(\gamma_{a c}-\gamma_{a b}-2 i \delta_{c}\right) \hbar}{2 \hbar} \frac{d}{d t} x_{a b}(t)+ \\
& {\left[x_{a b} \frac{W_{b c} W_{c b}}{4}+\frac{2 \Delta+\left[\left(-i \gamma_{a c}+2 \delta_{a}-2 \delta_{c}\right) \hbar\right]}{4 \hbar} \rho_{a a}^{(0)} W_{a b}\right] e^{\frac{t}{2}\left(\gamma_{a b}+i\left[\Delta+2 \delta_{a} \hbar\right] / \hbar\right)}=0 . }
\end{aligned}
$$
matrix:

Partial solution of this equation makes it possible to write a similar solution for $\rho_{a b}(t)$

$$
\rho_{a b}(t)=e^{i t\left[\Delta+2 \delta_{a} \hbar\right] / 2 \hbar} \frac{\left(2 \Delta+\left(-i \gamma_{a c}+2 \delta_{a}-2 \delta_{c}\right) \hbar\right)}{2 \hbar} \rho_{a a}^{(0)} W_{a b} U,
$$

where $U$ is a square matrix having the same order as the density matrix of the upper level $\left(\rho_{b b}(t)\right.$, in the case under consideration), that contains $I_{b}$ - the identity matrix of the same order as $\rho_{b b}(t)$ :

$$
U=\left[\frac{1}{4} W_{b c} W_{c b}-I_{b} \frac{\left(\Delta-i \gamma_{a b} \hbar+2 \delta_{a} \hbar\right)\left(2 \Delta+\left(-i \gamma_{a c}+2 \delta_{a}-2 \delta_{c}\right) \hbar\right)}{4 \hbar^{2}}\right]^{-1} .
$$

From expression (13), it is apparent that in the absence of relaxation processes, the difference of the frequency mismatches in each arm of the $\Lambda$-scheme is equal to the lower levels splitting of $\delta_{c}-\delta_{a}=\Delta$, and the absorption of the probe field in the $b c$ arm vanishing. This effect is known as electromagnetically induced transparency (EIT).

Based on formula (13), the expression for density matrix that defines the "low-frequency coherence" can be obtained:

$$
\rho_{a c}(t)=\frac{1}{4}\left(-e^{t \gamma_{a c} / 2}+e^{i t\left[\Delta+\left(\delta_{a}-\delta_{c}\right) \hbar\right] / \hbar}\right) \rho_{a a}^{(0)} W_{a b} U W_{b c}\left(1-e^{i t\left[\Delta+\left(\delta_{a}-\delta_{c}\right) \hbar\right] / \hbar}\right),
$$

as well as elements of the density matrix $\rho_{b c}(t)$, that determines coherence in $b c$ arm:

$$
\begin{aligned}
\rho_{b c}(t)=\left\{\frac { \hbar } { 4 } \left[\frac{\left(e^{-\gamma_{b c} t / 2}-e^{t\left(i \Delta+\left(\gamma_{a c}+2 i \delta_{a}\right) \hbar\right) / 2 \hbar}\right)}{\Delta+\left(-i \gamma_{a c}+i \gamma_{b c}+2 \delta_{a}\right) \hbar}+\right.\right. \\
\\
\left.\left.\quad \frac{\left(e^{-t \gamma_{b c} / 2}-e^{i t\left(\Delta-2 \delta_{c} \hbar\right) / 2 \hbar}\right)}{4\left(\Delta-i \gamma_{b c} \hbar+2 \delta_{c} \hbar\right)}\right] W_{b a} \rho_{a a}^{(0)} W_{a b} U W_{b c}\right\}-\frac{i}{2} e^{i t\left(\Delta-2 \delta_{c} \hbar\right) / 2 \hbar} W_{b c} \rho_{c c}^{(0)} .
\end{aligned}
$$




\section{Discussion on the subject of numerical calculation results.}

Before considering the items related to the solution of the system (9), some principal problems connecting to the possibility of laser generation should be discussed.

First of all, it should be noted that, as numerical calculation has demonstrated, the low-frequency coherence does not have a significant influence on the generation process. Much more significant impediments to the generation process are resonance collisions of excited atoms with atoms in ground state, that exhibit a destructive behavior, resulting in decay of generation.

From the theory of collisional depolarization [14], it is well-known that within the limits of the impact-parameter method, the relaxation constant of collisional process of two heavy particles, being described by the mutual interaction law $W \sim 1 / R^{n}$ (here $R$ is an internuclear distance), is described by the relaxation constant:

$$
\gamma=n_{0} v\left(\frac{Q}{\hbar v}\right)^{\frac{2}{n-1}} F(n),
$$

where $n_{0}$ is a projectile density (the density of the of buffer gas particles), $v$ - relative velocity of colliding particles, while $Q$ is a constant that depends on angular moments and interaction low, $F(n)$ is an expression that appears due to averaging of variation of the products of the density matrix over the impact parameter. Concerning the $Q$ quantity, it should be noted that this value is equal to the Van der Waals constants difference in the case of interaction by law $W \sim 1 / R^{6}$, and to the quadrupole moment in the case of interaction by law $W \sim 1 / R^{3}$. The first of these laws is implemented at interaction of two different atoms, whereas the second is utilized for the interaction of identical atoms. Owing to the fact that in coherent excitation experiments, the hyperfine sublevels of the ground state are usually used as the lower levels, then the influence of the collisions on those atoms is described by the law $W \sim 1 / R^{3}$, whereas influence of buffer gas on the excited state is described by law $W \sim 1 / R^{6}$. It follows from what was said, that the resonance collisions (collisions of two identical particles) leads to significant broadening of the lower hyperfine levels, and even to their overlapping.

Within the work under consideration, when lower levels, having a fine structure for the excited state with splitting of about $0.1 \mathrm{~cm}^{-1}$ are used [16], as a consequence, the influence of collisions with particles of buffer gas is fairly insignificant. In support of this statement, the following could be noted. On the one hand, for successful laser emission recording, it is necessary to induce significant impact population of the $2^{3} \mathrm{P}$ state of helium atoms ensemble. Without going into technical difficulties, it should be noted that as soon as such an ensemble has been created, atoms engaged in $2^{3} \mathrm{P}$ state would interact with each other by law $1 / R^{3}$, while with atoms of buffer gas by law $1 / R^{6}$. But it is clear that at given general density of helium atoms, the density of impact-excited atoms would be significantly less than the density of nonexcited particles, and therefore $2^{3} \mathrm{P}$ state of helium atom would be broadened to a significantly weaker extent. It should be noted as well that, in the case under consideration, one of the lower states $2^{3} \mathrm{P}_{2}$ is metastable [16], whereas the influence of finiteness of the lifetime of the second lower state $2^{3} \mathrm{P}_{1}$ can be taken into account by means of introducing the appropriate relaxation constants.

System (9) was integrated numerically, using method that has been described, in some detail, in our previous work [17]. On the system integration, we assumed that, at the initial point of time, the upper level is not populated $\rho_{i, b}(0)=0(i=a, b, c)$, whereas a numerical vales of the rest blocks of the density matrix are defined by formula (1). As for the fields that are active in the arms of $\Lambda$-scheme, in each arm, they are equal to the vector sum of the two fields: the stationary pumping field and the polarization one. With regard to the stationary 
pumping fields, we will assume that they are equal to zero at $t \leq 0$, and reach their preset values in a stepwise manner at $t>0$. Additionally, we will assume that at the initial point of time, the system undergoes a short "start-up" impulse, and later, at $t>0$, the field active in the system is defined by system (9). Numerical calculation results are represented in Figs. $2-6$.

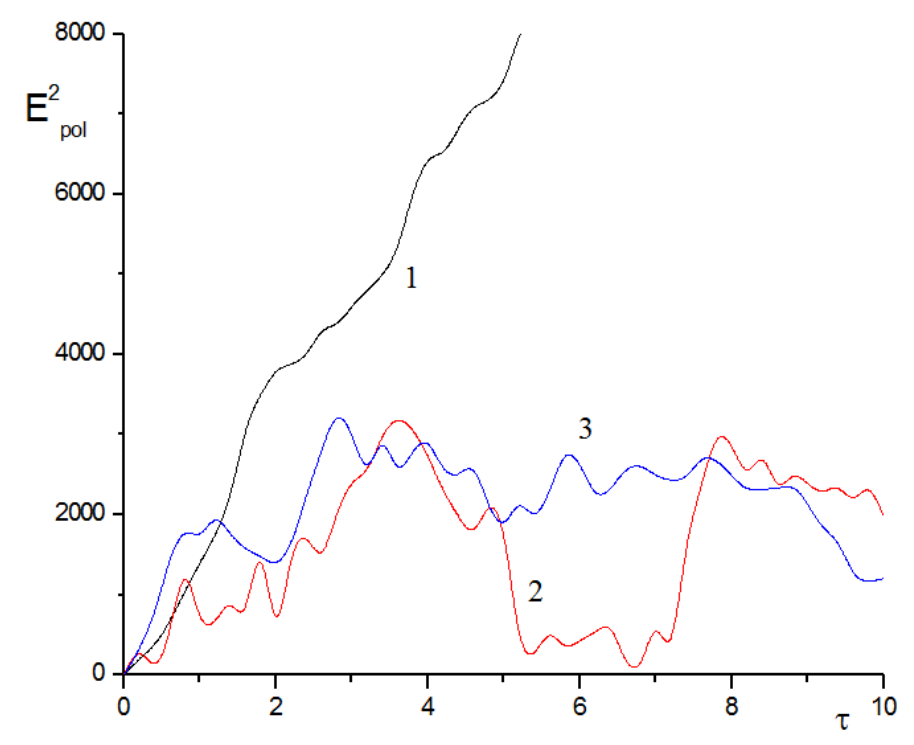

FIG. 2. The dependence of intensity of polarization field on dimensionless time. Curves 1-3 coincide the mismatches in $b c$ arm of $\Lambda$-scheme equal to $\delta_{c}=0$, $\Delta / 2, \Delta$ whereas mismatch in $b a$ arm is equal to zero

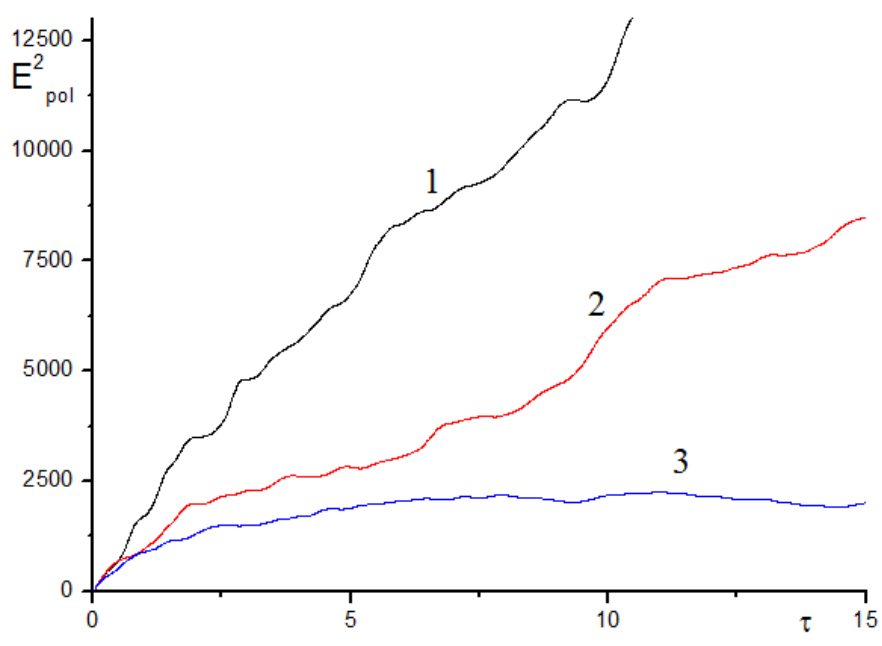

FIG. 3. The dependence of intensity of polarization field on the efficiency of excitation. Curves $1-3$ coincide to values of angle $\Theta=0, \pi / 4, \pi / 2$

In Fig. 2, the intensity of the polarization field for dimensionless time is represented for several mismatched dimensionless laser frequency values in the $b c$ arm: $\delta_{c}=0, \Delta / 2, \Delta$ whereas in another arm, the mismatch is constant: $\delta_{a}=0$. To that end, relaxation constants in the arms have been taken into account, according to the tabular values of transition probability $3^{3} \mathrm{~S}_{1} \rightarrow 2^{3} \mathrm{P}$ [16], with weights proportional to multiplicity of the above-mentioned states. It should be noted that the relaxation constants for the $2^{3} \mathrm{P}_{j}$ states, both in this figure and 


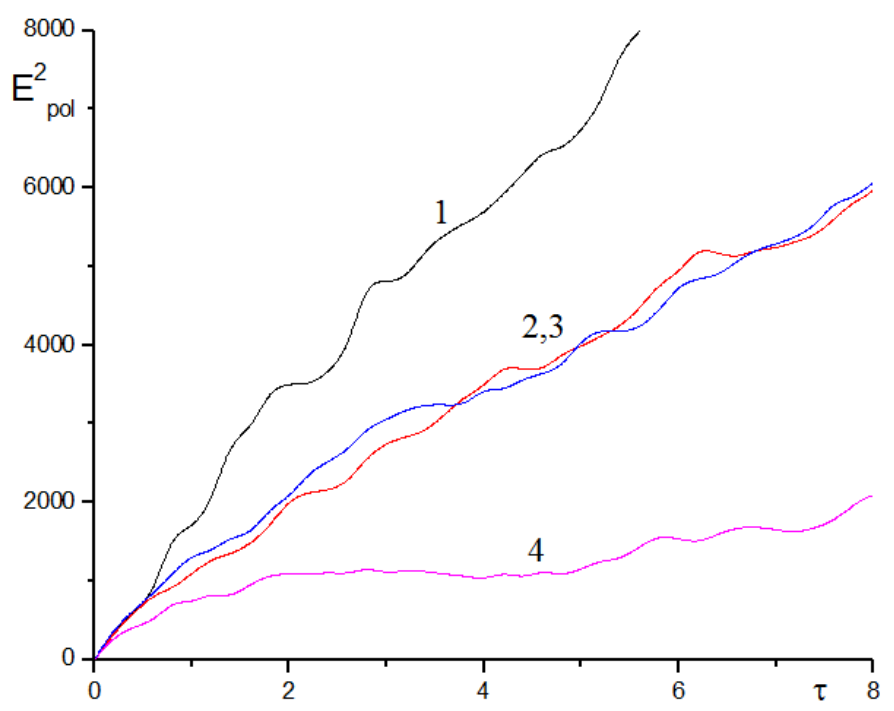

FIG. 4. The influence of relaxation on the generated field. Curve 1 is the curve 1 from the Fig. 1 (lower levels dose not relax); curve 2: each of levels "a" and "c" relaxed with the relaxation constant equal to the half of the spectroscopic value; curve 3: levels $2^{3} \mathrm{P}_{2}$ did not relax and $2^{3} \mathrm{P}_{1}$ relaxes with the constant equal to the spectroscopic value; curve 4: both lower levels relaxes with the rate constant equal to one-and-a-half time exceeding the spectroscopic value

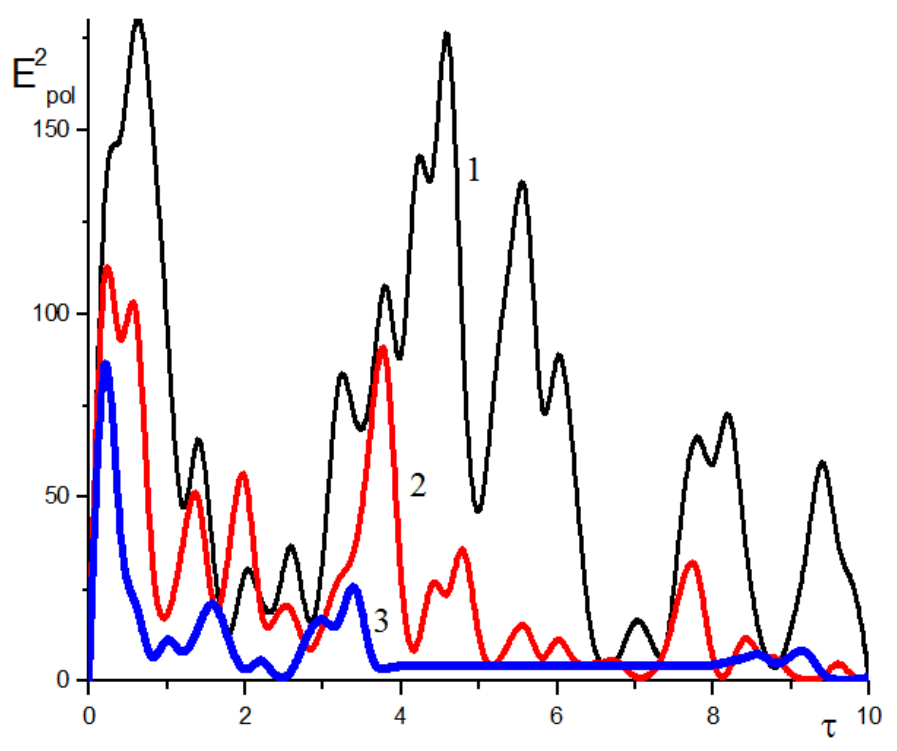

FIG. 5. All of the three levels, $3^{3} \mathrm{~S}_{1}, 2^{2} \mathrm{P}_{1}$ and $2^{2} \mathrm{P}_{2}$, are relaxing, with equally large relaxation constants $\gamma=4,6,8$ (curves $1-3$ consequently)

in subsequent ones, were assumed to be zero. It is apparent from this figure that emission represents a pulse train, with a magnitude that decreases as $\delta_{c}$ increases.

Figure 3 illustrates the dependance of the polarization field intensity on the efficiency of the lower levels population, that is characterized by angle $\Theta$ (see formula (1)). The calculation results show that the polarization field values are practically symmetrical with respect to the angle value $\Theta=\pi / 2$. Therefore, this figure depicts the field-angle dependence, for $\Theta=0$, 
$\pi / 4, \pi / 2$. It is apparent from the figure that for decreasing angle $\Theta$ within the preset limits, the polarization field intensity also decreases.

Subsequent figures illustrate the influence of relaxation on the generated field. It is worth noting that, in the case under consideration, the $2^{2} P$ state consists of two levels, $2^{2} \mathrm{P}_{1}$ and $2^{2} \mathrm{P}_{2}$, the latter of which is the metastable one.

In Fig. 4, four curves are presented that illustrate the dependance of the polarization field intensity on dimensionless time. The first of these curves takes into account the radiative decay of the upper level $3^{3} \mathrm{~S}_{1}$ on the lower levels $2^{2} \mathrm{P}_{1}$ and $2^{2} \mathrm{P}_{2}$, with the constants that correspond to the spectroscopic values [16] (curve (1) from the previous figure). Curves 2 and 3, in addition to the relaxation process of the upper state, take into account the radiative decay from the lower states, and at that, when plotting curve 2 , it has been assumed that each of $2^{2} \mathrm{P}$ levels decay at a rate equal to half that of the spectroscopic value, whereas curve 3 illustrates the case when level $2^{2} \mathrm{P}_{1}$ decays at a rate equal to the spectroscopic value, whereas the metastable level does not relax. Curve 4 describes the case when both of $2 \mathrm{P}$ levels relax at a rate 1.5 times that of the spectroscopic value. It is apparent from this figure that curves 2 and 3 practically identical, while further increase of the relaxation rate results in a decrease in the polarization field intensity.

In Fig. 5, the case was illustrated when all three levels, $3^{3} \mathrm{~S}_{1}, 2^{2} \mathrm{P}_{1}$ and $2^{2} \mathrm{P}_{2}$, are radiatively relaxing, with equally sized relaxation constants $\gamma=4,6,8$. It is apparent from the Fig. 4 that, for the last two relaxation constant values, emission consists practically of one pulse, and this is in agreement with the results of previous work [13].

In the conclusion of this paragraph, we will provide some approximate formulas that explain the appearance of the generated signal, and, particularly, the fact that the strongest generation corresponds to the mismatch $\delta_{c}=0$, provided that in another arm of the $\Lambda$-scheme there is no mismatch $\delta_{a}=0$ (see Fig. 2). To obtain these results, we used the software package Mathematica, deriving density matrixes values $\rho_{a a}(0), \rho_{c c}(0)$ (formula (1)), at the angle value $\Theta=0$, and after that, correlation matrices elements $\rho_{a b}$ and $\rho_{c b}$ were calculated analytically with help of (13) and (16) formulas. Based on those matrices, spurs of matrices $\operatorname{Sp}\left(r_{a b} \rho_{a a}(0) r_{a b} U\right)$, $\operatorname{Sp}\left(r_{b a} \rho_{a a}(0) r_{a b} U r_{b c} r_{c b}\right)$ and $\operatorname{Sp}\left(\rho_{c c}(0) r_{c b}\right)$, were calculated analytically, making it possible to obtain the right side of equation defining the polarization field:

$$
\frac{d}{d t} E_{p o l}=k E_{p o l} \operatorname{Sp}\left(\rho_{b c} r_{c b}+\rho_{b a} r_{b a}\right) .
$$

Then, the variable $x$ being imposed according to the relationship $\delta_{c}=x \Delta$ and assuming $\Delta$ to be large, in first order perturbation theory, the expression for the polarization field can be obtained :

$$
\ln \left(E_{p o l}\right) \sim \frac{(200-7 \sqrt{70}) e^{\frac{1}{2} i t(1-2 x) \Delta}}{4500(-1+2 x) \Delta} .
$$

From this formula, it is apparent that the maximum of polarization field is achievable at $x=0$ i.e. $\delta_{c}=0$, which coincides with the data in Fig. 2 .

\section{Conclusion}

In this work, the possibility of laser generation without inversion of the fine levels of helium atom is considered. It should be mentioned that considerations of the problem in this work and in articles quoted above [11,12] differ in principle. In the first part of this article, the positiveness of the reinforcement coefficient is achieved via radiation transitions from a specially-populated additional level, whereas in the second part of the paper, generation occurs 
in that arm of the $\Lambda$-scheme where the absorption is absent, due to the system achieving an EIT state.

A peculiar feature of this work consists in that for lower levels, used for the coherent excitation according to $\Lambda$-scheme, the excited states $2^{3} \mathrm{P}_{1}$ and $2^{3} \mathrm{P}_{2}$ of the He atom were used. The latter circumstances have permitted us to avoid significant broadening of the lower levels, by means of their interaction with buffer gas. At the same time, the creation and maintenance of a sufficient population at those levels is most probably caused by a strain to the system.

\section{Acknowledgments}

This work was partially financially supported by the Government of the Russian Federation (grant 074U01), by Ministry of Science and Education of the Russian Federation (GOSZADANIE 2014/190, Projects No. 4.Z50.31.0031 and No. 1.754.2014/K), by grant 1611-10330 of Russian Science Foundation.

\section{References}

[1] Kocharovskaya O., Hanin Ia.I. Coherent amplification of the ultra shot impuls in the three level madium without the population iunversion. Letters to JETP, 1988, 48, P. 581-583.

[2] Harris S.E. Lasers without inversion: Interference of lifetime-broadering resonsnce. Phys. Rev. Lett., 1989, 62(9), P. 1033-1036.

[3] Imamoglu A., Harris S. Lasers without inversion: interference of thr dressed lifetime-broadened states. Opt. Lett., 1989, 14(24), P. 1033-1036.

[4] Scully M.O., Zubairy M.S. Ouantum Optics, Cambridge University Press, 1997.

[5] Kocharovskaya O., Mandel P. Amplidication without inversion: The double $\Lambda$-shime. Phys. Rev. A, 1990, 42(1), P. 523-535.

[6] Harris S.E., Field J.E., Imamoglu A. Nonlinear optical processes using electromagnetically induced transparency. Phys. Rev. Lett., 1990, 64(10), P. 1107-1110.

[7] Scully M. Phys. Reports. From lasers and masers to phaseonium ahd phasers. 1992, 219, P. 191-202.

[8] Kocharovsaya O.A. From lasers without inversion to grasers? Laser Phys., 1995, 5(2), P. 284-291.

[9] Kocharovsaya O.A. Amplification and lasing without inversion. Phys. Reports, 1992, 219, P. 175-190.

[10] Kocharovsaya O.A., Kolesov R., Rostovtsev Yu. Lasing without inversion: a new path to gamma-ray laser. Laser Phys., 1999, 9(4), P. 745-753.

[11] Zibrov A.S., Lukin M.D., et al. Experimental demonstration of laser oscillation without population inversion via quantum interference in Rb. Phys. Rev. Lett., 1995, 75(8), P. 1499-1501.

[12] Pandanabandu G.G., Welch G.R., et al. Laser oscillation without population inversion in sodiul laser beam. Phys. Rev. Lett., 76(12), P. 2053-2056.

[13] Ni Cui, Christof C.H., Macovei M. Interference-induced peak splitting in extreme ultraviolet superfluorescence. Optics Lett., 2013, 38(4), P. 570-572.

[14] Kazantsev S.A., Petrashen A.G., Firstova N.M. Impact spectropolarimetric sensing, Kiuwer Academic/Plenum Publishes, 1999.

[15] Jucys A., Bandzaitis A.A. Theory of the momentum in quantum mechsnics, Vilnius 1965, $462 \mathrm{p}$.

[16] Radzig A.A., Smirnov B.M. Reference data on atoms, molecules and ions, Springer Series in Chemical Physics, Berlin, 1985.

[17] Petrashen A.G., Sytenko N.V. Polarization characteristics of radiation of atomic ensamble under coherent exitation in the presrnce of the strong magnetic field. Nanosist.: Phys., Chem., Math., 2015, 6(1), P. 122-132. 